\title{
AUTOMATED IMAGE ANALYSIS OF NOISY MICROARRAYS
}

\author{
Sharon Greenblum, Max Krucoff \\ Department of Biomedical Engineering, Northwestern University, Evanston, IL, USA \\ s-greenblum@northwestern.edu,m-krucoff@northwestern.edu \\ Jacob Furst, Daniela Raicu \\ School of Computer Science, Telecommunications, and Information Systems, DePaul University, Chicago, IL, USA \\ jfurst@cti.depaul.edu,draicu@cti.depaul.edu
}

Keywords: DNA Microarray, image analysis, noise, segmentation, gridding, quantification, addressing, indexing.

\begin{abstract}
A recent extension of DNA microarray technology has been its use in DNA fingerprinting. Our research involved developing an algorithm that automatically analyzes microarray images by extracting useful information while ignoring the large amounts of noise. Our data set consisted of slides generated from DNA strands of 24 different cultures of anthrax from isolated locations (all the same strain that differ only in origin-specific neutral mutations). The data set was provided by Argonne National Laboratories in Illinois. Here we present a fully automated method that classifies these isolates at least as well as the published AMIA (Automated Microarray Image Analysis) Toolbox for MATLAB with virtually no required user interaction or external information, greatly increasing efficiency of the image analysis.
\end{abstract}

\section{INTRODUCTION}

In the field of genetic analysis, DNA microarrays have become a go-to method for studying gene expression in an organism by measuring the ratios of multi-channel hybridization. A recent extension of this technology, however, is its use in DNA fingerprinting, i.e. generating a unique pattern of probe hybridization for an unknown DNA sequence to compare with known DNA sequences and identify its origin. This less-explored avenue of genetic analysis has led to new challenges in the area of microarray image processing, for which few techniques have been developed.

Of the existing programs (for example, the AMIA Toolbox for MATLAB (White, 2005)), none are fully automated. A non-automated program may require a sizeable amount of user input regarding spot size, seeded region growing thresholds, array size, control point size and location, and starting points for grid creation. The necessity of manually entering this information requires more background knowledge of the slide than may be available, influences the image processing depending on the user running the program, and significantly slows down the overall time required to analyze a slide.

In light of these inefficiencies and shortcomings, we present a new, fully-automated image processing method for grayscale intensity microarray images. In addition, we accommodate slides with extremely low signal to noise ratios (SNRs). Our data set consisted of slides generated from DNA strands of 24 different cultures of anthrax from isolated locations (all the same strain). Each isolate contained 9 slides, each of which had four $10 \times 10$ spot arrays. In total, we analyzed $86410 \times 10$ spot arrays on 216 separate slide images. The data set was provided by Argonne National Laboratories in Illinois.

\section{BACKGROUND}

Many microarray image processing techniques exist that attempt to extract useful information from images while ignoring background noise. Most techniques divide the process into three steps: gridding (addressing each spot), segmentation (separating spot pixels from background pixels), 
and quantification (putting spot intensity data into numerical form for comparison).

\subsection{Gridding}

Because it is often easiest to analyze each $10 \times 10$ array separately, 'super' or 'global' gridding is needed. This is the process of separating each array into its own image. Once this is achieved, the dots themselves can be gridded within the supergridded array. This provides an index (or address) for each dot (or lack thereof).

There are a number of challenges associated with both supergridding and gridding. For example, individual dots may be translated from a regular array pattern due to bent or otherwise offcenter dipping pins used to create the dots. Furthermore, some dots in a microarray image may have very weak (or absent) intensities and may be hard to detect. Finally, noise in the image due to elements of the image capturing techniques (e.g. washing techniques, dust, scratches, etc.) may interfere with gridding algorithms.

In an attempt to tackle these challenges, various gridding methods have been employed including manual gridding, horizontal and vertical profiling (Blekas, 2005), a Bayesian approach to deforming a regular grid (Lipori, 2005; Ho, 2006), and a Markov random field based approach (Katzer, 2003).

\subsection{Segmentation}

Once a spot's location is known, separating the dot pixels from the background pixels provides another challenge. This process can be difficult due to inconsistent background intensities within one image as well as across many slides due to smudges, overlap of extremely bright dots, and variation in washing techniques. In addition, spot morphology is rarely consistent and the location of a dot within a grid box can vary considerably. Finally, weak dots can be very hard to distinguish from a noisy background, even visually.

Methods that have been proposed to confront these challenges include a Hough transform to find circles (Horsthemke, 2006), K-means clustering $(\mathrm{Wu}, 2003)$ of pixels within a grid box, fixed or adaptive circle segmentation (Yang, 2001), adaptive ellipse methods (Rueda, 2005), adaptive shape methods (using watershed or seeded region growing) (Yang, 2001; Angulo, 2003), histogram segmentation (Yang, 2001), and Gauss-Laguerre wavelets to create an enhanced image that can be used as a mask (Pallavaram, 2004).

\subsection{Quantification}

The ultimate goal of image processing is to obtain values representative of spot intensities so that the degree of DNA hybridization can be analyzed and compared.

Proposed methods of addressing this challenge include simply averaging all foreground pixel intensities, averaging foreground pixels and subtracting or dividing by a local or global background intensity, fitting of a parametric model to pixel intensities with the help of M-estimators (Brändle, 2003) and integrating individual pixel intensities to obtain a spot intensity reading (Bemis).

\section{METHODS}

When attempting to analyze real (non-ideal) microarray images, large amounts of noise can confound automatic algorithms. Therefore, it is necessary to first eliminate this noise before proceeding with further analysis. Generally speaking, the noise inherent in these images, while differing from image to image, has certain specific properties that enable us to differentiate it from the signal. Many steps in our procedure check for these properties and use them to filter out the noise.

\subsection{Addressing/Indexing}

\subsubsection{Supergridding}

Orientation spots were used to separate the full slide into smaller and more predictable grids. Orientation spots are intended to be the brightest spots on the array and are used to make sure that a slide is not upside-down or in an incorrect orientation during image capture (Figure 1).

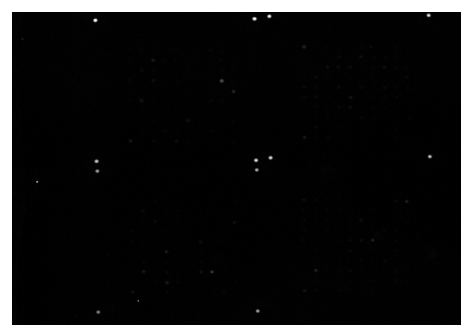


Figure 1: An original slide image as visualized in MATLAB. Only the orientation spots can be seen because of their relative brightness.

From here we use horizontal and vertical profiling to create a 'supergrid' that can be used to crop the image (Figure 2).

A

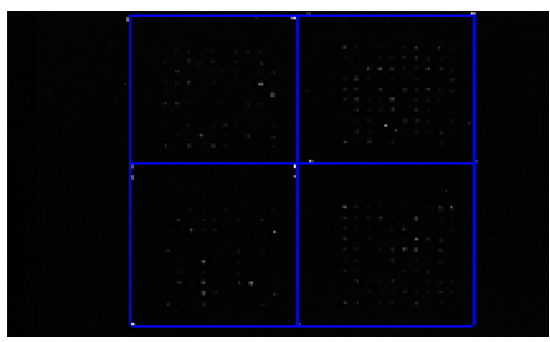

B

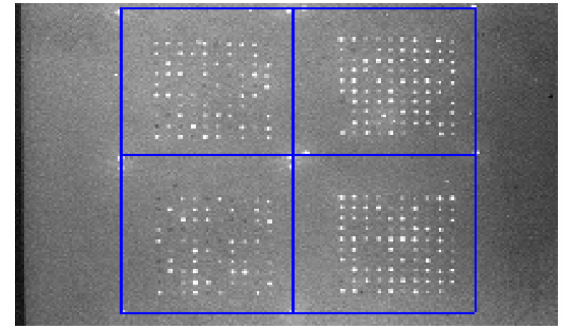

Figure 2: A) Supergrid drawn over original image. B) Supergrid shown over enhanced image. Now we can see the spots of interest and the four sections.

At this point, the image is cropped and each section is analyzed separately.

\subsubsection{Gridding}

After the original image is cropped into its four sections, our program grids each of the new images separately. Our process applies a sequence of filters to each image to ensure that any information used in the profiling is actual data. Then we apply a set of quality control loops that complete grids when data is missing and eliminate rows and columns when there is still noise included even after the filtering. In the gridding process, we are more concerned with eliminating false data than ignoring weak data because this ensures that we will get a more accurate grid. In segmentation, we look at the original, unfiltered image, so weak data will be included.

Our process begins by applying a median filter that helps eliminate salt and pepper noise (Figure 3). Next we apply a disc filter similar to that applied during supergridding (Figure 4).

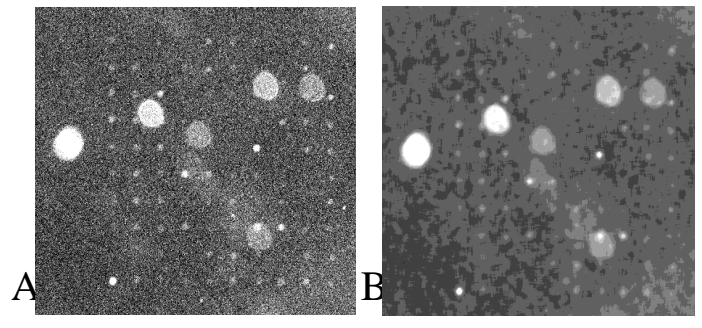

Figure 3: A) Enhanced view of original crop. Notice the salt and pepper noise. B) Same crop after median filter has been applied. There is much less randomness to the pixel values, and more structure has been introduced.

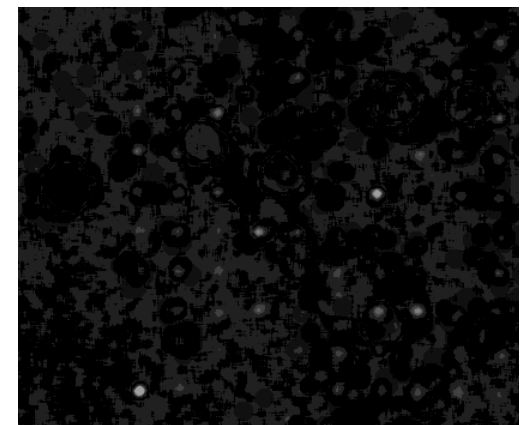

Figure 4: Disc filter applied to the image shown in figure 3B. Notice that the large splotches have been eliminated, as well as any uneven illumination.

From here, we convert the image to black and white using a thresholding technique, and the edges of each image are cropped to remove any remnants of the orientation spots still in the image (which are now treated as noise-Figure 5).

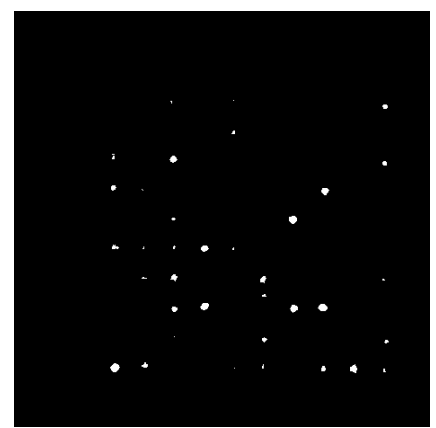

Figure 5: Image with cropping at the edges. Notice the deletions of potentially misleading data.

Since there may still be noise left in the image, we apply our novel filters next: a 'pixel filter' and an 'oblong filter' that remove, respectively, stray pixels and oblong shapes from the black and white image (Figure 6). 


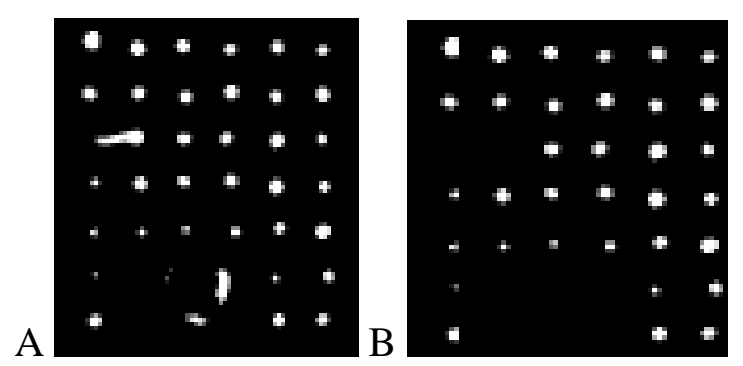

Figure 6: An example of the effectiveness of the oblong filter at removing non-circular data. A) Black and white. B) After oblong filter.

Now we can apply horizontal and vertical profiles to generate a preliminary grid of the data (Figure 7).

A

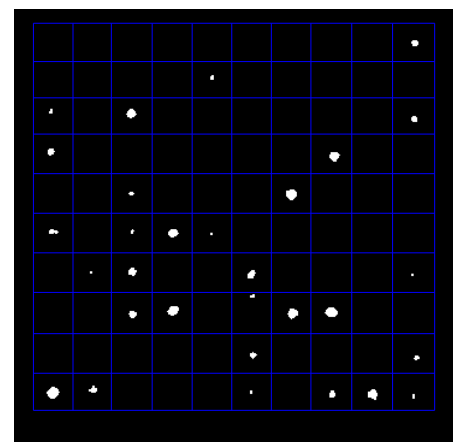

B

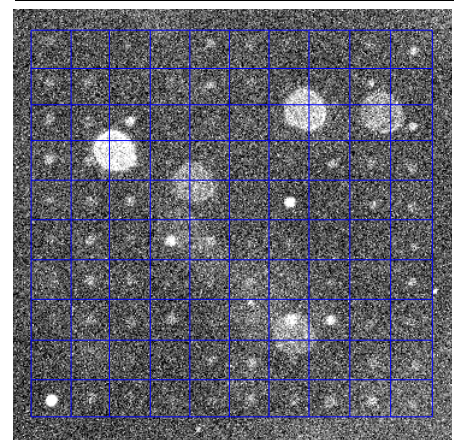

Figure 7: A) Grid shown over black and white image. B) Grid shown over original enhanced image from Figure 3. Notice how much noise it ignores.

Sometimes, especially when whole rows and/or columns are absent in the original image, our grid at this point is not satisfactory. From here, the image runs through our novel control loops that check for grid columns and rows that are too large and too small, as well as grids that have too many or too little rows and columns. The control loops then fill in missing information or delete extraneous information based on expected sizes of rows and columns within a certain range. If there is enough information in the slide, the control loops should not have to be used. However, in the cases in which whole rows or columns are missing, our automated program will fill them in. (Figure 8).
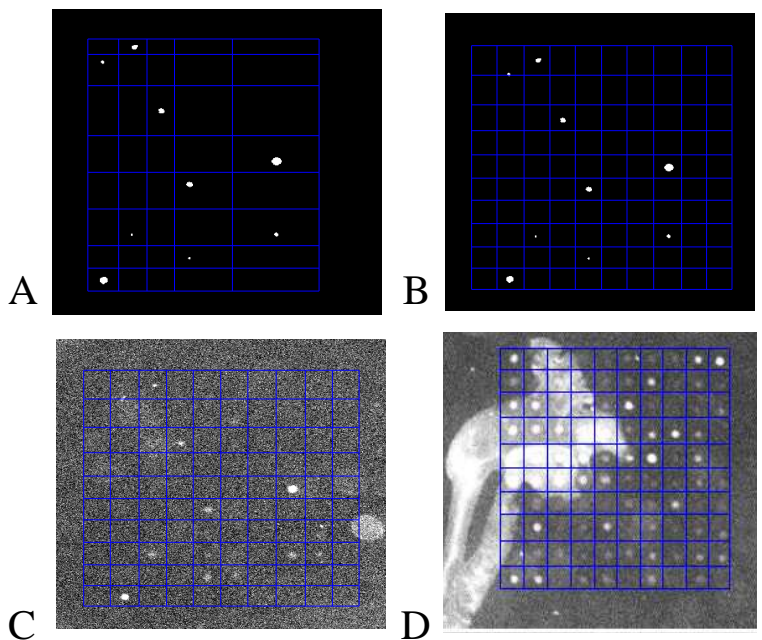

Figure 8: A) An example of a preliminary grid of a crop without much useful information. B) Same slide after it has run through our control loops. C) The final grid shown over an enhanced view of the original crop. D) Example of gridding results over noisy data.

\subsection{Spatial Segmentation}

Once the image has been correctly addressed, we would expect the spots to be approximately in the center of each grid box. Therefore, one approach to spatial segmentation is to use a "centered circle" scheme. In this technique, a circle of known diameter is drawn in the center of each grid box. All the pixels inside the circle are considered 'spot pixels,' and all the other pixels in the box are considered 'background pixels' (Figure 9). We use the original, unfiltered image for data collection.

Another approach to spatial segmentation is to use a 'wandering circle' method. In this procedure, our program takes a circle of expected spot diameter and moves it throughout a specified area within each grid box, searching for the maximum average intensity. It uses this location as the spot location (Figure 9). Again, we use the original, unfiltered image for actual data collection.

A
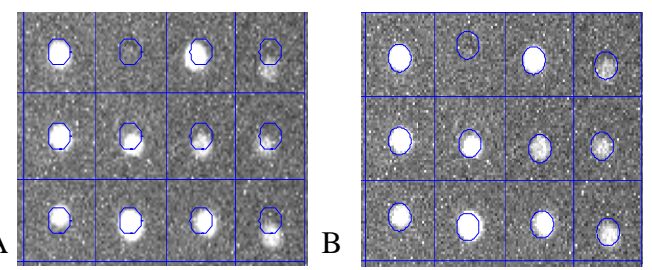
Figure 9: A) A close up view of the centered circle approach and B) the wandering circle approach.

\section{RESULTS}

We compared our results to that of MATLAB's AMIA (Automated Microarray Image Analysis) toolbox. The classification results were generated using a Support Vector Machine and 9-fold crossvalidation of the data.

The centered circles approach worked the best; the gridding correction step added a small boost to the accuracy (total number of correct classifications divided by the total number of replicates). The results are shown below:

\section{Percent of Isolates Classified Correctly \\ Centered Circles alone: $56.28 \%$ \\ Grid Corrections: $56.68 \%$ \\ AMIA: $55.35 \%$}

The generally low percentages may be due largely to the poor quality of the images and the very close similarities between the strands, not necessarily the image processing techniques. It also may have to do with the applied statistical methods.

Possible improvements to these results are discussed in the future work section below.

\section{CONCLUSIONS AND FUTURE WORK}

Because we found a method with at least equal accuracy and greater automation than the AMIA toolbox, we consider our work an improvement on DNA microarray image processing for grayscale intensity, noise-filled image classification. The only user input required for our program to run all the way through is for the user to locate the folder in the computer that contains the images. It was surprising to see that the wandering circle method did not improve upon the centered circle method. One reason for this inconsistency might be that noise has too great an effect on circle location.

We will also investigate different statistical approaches - the literature has shown techniques that generate almost $90 \%$ accuracy on the AMIA data, and we feel that more advanced statistical analyses will generate even better results on data generated by our algorithms.

\section{REFERENCES}

White, A., Daly, D., Willse, A., Protic M., and Chandler, D., 2005. "Automated microarray image analysis toolbox for MATLAB." Bioinformatics 21:3578-3579.

Blekas, K., Galatsanos, N., Likas, A., Lagaris, I., 2005. "Mixture model analysis of DNA microarray images." IEEE Trans. Med. Imaging 24(7): 901909.

Lipori, G., 2005. "Efficient gridding of real microarray images," Proceedings of the Workshop on Biosignal Processing and Classification of the International Conference on Informatics in Control, Automation and Robotics.

Ho, J., Hwang, W., Horn-Shing Lu, H., Lee, D., 2006. "Gridding Spot Centers of Smoothly Distorted Microarray Images" IEEE Transactions on Image Processing.

Katzer, M., Kummert, F., Sagerer, G., 2003. "A Markov random field model of microarray gridding," Symposium on Applied Computing, pp. 72-77.

Horsthemke, B., Furst, J., 2006. "DNA Microarray Spot Detection Using Hough Transforms", CTI Research Symposium.

Wu S., Yan, H., 2003. "Microarray image processing based on clustering and morphological analysis," Proceedings of the First Asia-Pacific Bioinformatics Conference on Bioinformatics, Vol. 19, pp. 111-118.

Yang, Y., Buckley, M., Speed, T., 2005. "Analysis of cDNA microarray images," Briefings in Bioinformatics, Vol. 2, pp. 341-349.

Rueda, L., Qin, L., 2005. “A New Method for DNA Microarray Image Segmentation” International Conference on Image Analysis and Recognition, pp 886-893.

Angulo, J., Serra, J., 2003. "Automatic analysis of DNA microarray images using mathematical morphology," Bioinformatics 19(5): 553-562.

Pallavaram, Carli, M., Berger, J., Neri, A., Mitra, S., 2004. "Spot identification in microarray images using Gauss-Laguerre wavelets", Proc. IEEE Workshop on Genomic Signal Processing and Statistics.

Brändle, N., Bischof, H., and Lapp, H., 2003. "Robust DNA microarray image analysis," Machine Vision Applications.

Bemis, R., "DNA Microarray Image Processing Case Study," MATLAB Central. $<$ http://www.mathworks.com/matlabcentral/fileexc hange/loadFile.do?objectId=2573\&object Type=FIL E> 